BULLETIN Bulletin hispanique

HISPANIQUE Université Michel de Montaigne Bordeaux

117-2 | 2015

Métamorphose(s) : représentations et réécritures

\title{
Un vivo cadáver
}

la métamorphose du vivant en cadavre dans le théâtre de Calderón

Didier Souiller

\section{(2) OpenEdition}

Journals

Édition électronique

URL : http://journals.openedition.org/bulletinhispanique/4014

DOI : 10.4000/bulletinhispanique.4014

ISSN : $1775-3821$

Éditeur

Presses universitaires de Bordeaux

Édition imprimée

Date de publication : 15 décembre 2015

Pagination : $517-528$

ISBN : 979-10-300-0041-2

ISSN : 0007-4640

Référence électronique

Didier Souiller, « Un vivo cadáver », Bulletin hispanique [En ligne], 117-2 | 2015, mis en ligne le 15

décembre 2018, consulté le 19 avril 2019. URL : http://journals.openedition.org/

bulletinhispanique/4014; DOI : 10.4000/bulletinhispanique.4014 


\title{
Un vivo cadáver : la métamorphose du vivant en cadavre dans le théâtre de Calderón
}

\author{
Didier SOUILLER \\ Université de Bourgogne
}

Il y a un lien du théâtre de Calderón avec le fonctionnement de la vanité : la métamorphose opérée sur la scène détermine la même prise de conscience pour le spectateur, grâce au bouleversement que procure le surgissement de la mort, en assistant à une métamorphose spectaculaire; démonstration centrale, qui se retrouve dans différents genres et qui sous-tend même le recours à certaines figures de style privilégiées.

Mots-clés : Calderón, vanité, désabusement, écriture.

Hay una auténtica relación entre el teatro entero de Calderón y el empleo de las vanidades: la metamorfosis en las tablas produce una nueva conciencia de los espectadores que se dan cuenta de lo que significa el surgimiento de la muerte, cuando se ve una espectacular transformación; se trata de una demostración muy central que se encuentra también en los diferentes géneros dramáticos y que da su justificación a varios recursos estilísticos.

Palabras claves: Calderón, vanidades, desengaño, estilo.

A real link exists between Calderon's plays and the religious painting of a vanity: a metamorphosis occurs on the stage and gives way to the same type of consciousness in the mind of the audience -thank to the sudden appearance of death- by looking at a spectacular transformation; one can find this central demonstration in the diverse dramatic genres as well as at the origin of some of the writer's favourite tropes.

Keywords: Calderon, religious painting, disillusion, writing technique.

I A PRÉSENCE DU CRÂNE, représentation physique de la mort, occupe une L place de choix dans l'iconographie du Siècle d'Or, notamment dans le genre des vanités où tel est l'objet accompagnant invariablement les figures Bulletin Hispanique, Tome 117, $\mathrm{n}^{\circ} 2$ - décembre 2015 - p. 517-528. 
de saint Jérôme, saint François ou Marie-Madeleine, méditant sur un crâne pour symboliser cette étape essentielle du desengaño : la prise de conscience de la fragilité de la vie, l'illusion fugace des biens offerts par l'existence et la finitude de notre condition. Le desengaño tient traditionnellement un miroir ${ }^{1}$ pour marquer la nécessité d'un retour sur soi et de la contemplation de son image réelle. Dans le cadre de cet ascétisme teinté de néo-stoïcisme, qui prévaut au sein du discours religieux espagnol du Siècle d'Or et dans la mouvance de la Contre-Réforme (cf. le P. Nieremberg, Diferencia entre lo temporal y eterno, crisol de desengaños, Madrid, $1640^{2}$ ), le lien du théâtre avec le fonctionnement de la vanité apparaît clairement : la métamorphose opérée sur la scène doit déterminer, dans l'esprit du spectateur, la même prise de conscience que celle qui frappe le contemplateur d'un tableau où la présence évidente de l'horreur de la mort se trouve réunie aux objets et aux êtres qui incarnent les prestiges $\mathrm{du}$ monde : telles sont les innombrables illustrations du thème du finis gloriae mundi, dont le célèbre tableau de Valdés Leal (hôpital de la Charité, Séville) n'est qu'un exemple parmi bien d'autres.

En définitive, le théâtre, tirant profit de la pratique du symbolisme allégorique de l'auto sacramental, se révèle un mode d'expression privilégié pour donner à voir la présence de la mort et le bouleversement que son surgissement procure grâce à une métamorphose spectaculaire. Le goût de la métamorphose est d'ailleurs une caractéristique du théâtre " baroque " européen en général et de celui du Siècle d'Or en particulier : dans le cadre d'une esthétique qui privilégie le mouvement (sous toutes ses formes), il profite de la vogue des Métamorphoses d'Ovide, poète païen s'il en est, mais qui se trouve habilement réconcilié avec la métamorphose chrétienne dans une tentative de récupération des mythes antiques dont témoignent Juan Pérez de Moya (Philosophia secreta, donde debajo de historias fabulosas se contiene mucha doctrina provechosa a todos estudios. Con el origen de los idolos o Dioses de la Gentilidad, 1583) et Fray Baltasar de Vitoria (Teatro de los Dioses de la Gentilidad, 1620-1623). Calderón, au demeurant, dès El Sacro Parnaso (779b) prend soin de souligner le caractère licite du rapprochement, au cours d'une discussion de la Foi (catholique) avec Judaïsme et Paganisme (armé des Métamorphoses du poète latin) :

\author{
Bien veis cuánto en sus principios \\ Hebrea y latina frase \\ Convienen, simbolizadas \\ Fábulas y realidades.
}

En fait, si la métamorphose en cadavre occupe incontestablement le centre de quelques drames parmi les plus importants du dramaturge, cette

1. Voir El Gran mercado del mundo, 234b. En l'absence d'autre précision, toutes les références chiffrées renvoient à l'édition Aguilar en trois volumes des Obras completas de Calderón de la Barca, t. I, Dramas, 1969 ; t. III, Autos sacramentales, 1967, pour les premières éditions.

2. Voir Hugues Didier, Vida y Pensamiento de Juan E. Nieremberg, Madrid, Fundación Universitaria Española, 1976. 
transformation spectaculaire vient prendre place dans une démonstration centrale, qui se retrouve, peu ou prou, dans l'ensemble des œuvres à finalité religieuse (c'est-à-dire toutes celles dont le but n'est pas explicitement comique : du drama de santos à l'auto) et rend même compte de certaines figures de style caractéristiques de l'écriture caldéronienne.

Trois pièces accordent, en effet, une importance particulière à cette métamorphose. Un auto, datant probablement du début de la carrière de Calderón, El gran Duque de Gandía, s'appuie sur l'épisode de la conversion de François Borgia, futur général des jésuites, au spectacle de l'ouverture accidentelle du cercueil de la femme qu'il aimait ; l'impératrice s'est métamorphosée en un cadavre en décomposition, source d'une méditation désabusée qui s'exprime en oxymores devant le scandale de la mort et les antinomies de l'existence (108-109) :

\section{[...] porque otra viene a ser hoy de aquella que era ayer. en fin, en fin: fue hermosura esta espantosa fealdad, este estrago fue deidad, esta sombra lumbre pura, esta desdicha ventura [...].}

L'aventure du galant duc de Gandie sert de prétexte à une allégorisation de l'Homme, amoureux de Naturaleza, et que la métamorphose de ce qu'il aime convainc de la justesse de la réponse offerte par la Religion, où il trouvera la «Compagnie » qu'il recherche (110b) : si l'auto n'est pas sans lien avec la canonisation de Francisco de Borja en 1624 et sa béatification en 1671, il faut rappeler que Calderón fut l'élève des jésuites (Colegio imperial de Madrid).

Interprétation caldéronienne du mythe de Faust, El Mágico prodigioso présente un philosophe détourné de sa quête intellectuelle par le démon, qui profite de l'amour éprouvé par Cyprien pour la belle Justine afin de s'emparer de son âme, en échange de laquelle il lui livrera la jeune fille. Malheureusement pour le philosophe passionné, Justine est chrétienne et même si toute la nature se met à chanter les prestiges de l'amour, appuyée sur son libre-arbitre (inébranlable, 633b), celle-ci résiste victorieusement à la tentation diabolique : il ne reste plus au démon, maître de la magie et des apparences, qu’à offrir à Cyprien un succube qui prendra la forme de la belle Justine. Or, au moment où le personnage faustien s'empare du simulacre de la jeune fille, celui-ci lui échappe, se métamorphose en squelette et reprend spectaculairement le topos du finis gloriae mundi (636a) : 
De là, révolte contre le démon et appel au Dieu des chrétiens, dont le pouvoir supérieur s'est manifesté au cours de l'épisode, ce qui détermine un ultime renouvellement de la quête :

\author{
¡Grande Dios de los cristianos! \\ A ti en mis penas acudo ${ }^{3}[\ldots]$ \\ Más me ha de dar, pues le busco. (638)
}

La leçon de la vanité est explicitée lors du discours-confession devant le gouverneur : puisque toute chose est susceptible de se métamorphoser sous l'empire de la mort, seul Dieu peut donner un sens au monde et permettre d'échapper à la néantisation :

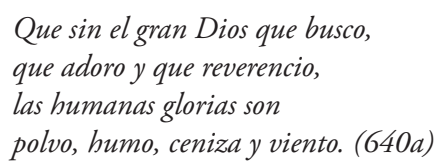

El Purgatorio de san Patricio : troisième et dernier exemple d'un épisode de métamorphose, qui constitue l'apogée d'une quête spirituelle et le signe suprême destiné à mettre fin à l'errance (tant physique qu'intellectuelle) du protagoniste, avant un nouveau départ vers une clarté, qui reste, pour tous, le point fixe entrevu parmi les tribulations les plus diverses ${ }^{4}$. Le héros du Purgatoire, Ludovico Enio, est le modèle parfait du révolté caldéronien, accumulant, non sans fierté, les crimes, comme il l'avoue, au cours de sa confession devant le roi :

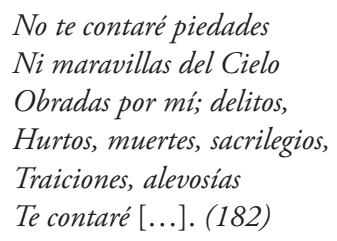

Un soir, revenu secrètement se venger d'un ennemi qui l'a offensé, Ludovico se trouve empêché par un mystérieux cavalier masqué ; exaspéré, le héros lui arrache son manteau et découvre un squelette qui s’adresse à lui :

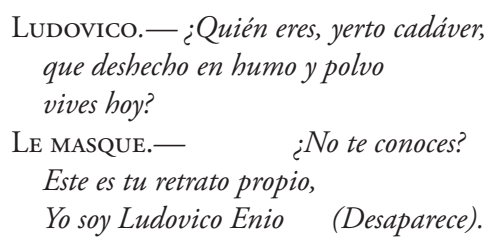

3. Le verbe acudir employé ici rappelle l'expression Acudamos a lo eterno (530b), qui accompagne la conversion de Sigismond dans La Vie est un songe, conversion qui fait suite à une métamorphose, purement intellectuelle cette fois.

4. " [J]'irai rejoindre cette sphère [divine] dont je poursuis les rayons ", Prince constant, 259b. Traduction de l'auteur. 
En un sens, cette rencontre avec son double métamorphosé en squelette, fonctionne comme le miroir tendu par le desengaño ; le protagoniste, au spectacle de ce qu'il est en réalité (un cadavre vivant), se métamorphose à son tour en repenti exemplaire par une sorte de nouvelle naissance :

\author{
[...] puedo alcanzar perdón, \\ Cuando arrepentido lloro. \\ Yo lo estoy, señor, y en prueba \\ De que hoy empiezo a ser otro, \\ $Y$ que nazco nuevamente, \\ En vuestras manos me pongo. (203 a)
}

Étant donnée la finalité didactique de cette vanité transposée sur la scène, les autos, bien évidemment, s'emparent du motif de la métamorphose, puisque, conformément au prologue Au lecteur de l'édition des Autos sacramentales y alegóricos de don Pedro Calderón de la Barca (Madrid, 1677), il ne s'agit, au travers d' "arguments " toujours variés, que d'un seul et même "sujet " : le dangereux pèlerinage de l'existence, jalonné d'expériences qui conditionnent la possibilité du salut. Au sein de la représentation allégorique de la vie, l'expérience-clef sera donc la rencontre avec la mort, surgie de la métamorphose des vains objets du "divertissement ", au sens pascalien, auxquels l'Homme s'est attaché. Les exemples sont nombreux ; aussi a-t-il semblé préférable de s'attacher à ceux qui mettent en valeur le cheminement dialectique de l'auto dans lequel vient s'intégrer la métamorphose en cadavre.

Dans El Pleito matrimonial, la mise en scène du conflit de l'âme et du corps, condamnés à vivre ensemble, malgré un destin différent, permet, d'emblée, de souligner la puissance de la mort sur le Corps : dès les premiers pas de celui-ci, il trébuche et tombe dans les bras de la Mort (79a). Quand le Corps agonise, il voit sa Vie s'éteindre, sous la forme d'une torche que souffle la Mort, avant d'être conduit dans la grotte de la Terre :

\author{
[...] Duro seno, \\ Desnudo de ti salí \\ $Y$ asi a ti desnudo vuelvo. \\ En los brazos de la Muerte \\ Naci y en sus brazos muero. (91b)
}

À plusieurs reprises, le devenir du Corps est remplacé, avec la même valeur démonstrative, par la Beauté (Hermosura) dont la fortune est, elle aussi, inséparable de la Mort. Dans No hay más fortuna que Dios, Hermosura, "ciega en sus divertimientos, / En una sima ha caído"; en sort un squelette, qui annonce à ceux qui demeurent sur la scène qu'il ne leur reste que : "humo, polvo, nada y viento" $"(630-631)$.

Si la vie est énigme, deux épisodes analogues cherchent à en fournir la clef. Dans Sueños hay que verdad son, le dramaturge reprend l'épisode biblique de Joseph expliquant le songe de Pharaon des vaches grasses et des vaches maigres; 
le prophète commence par insister sur le pouvoir du temps, qui conduit, inéluctablement, au néant :

$Y$ es el tiempo también el que, inconstante,

Todo lo deja a nada reducido. (1222b)

Pareillement, dans La Cena del rey Baltasar, une fois les trois mots fatidiques inscrits sur le mur et expliqués par Daniel, le roi Baltasar, au milieu de ses concubines et des Grands de son royaume, doit se battre avec la Mort venue, déguisée, assister au divertissement du festin royal : puisque "nulla est redemptio " (176a), c'est elle qui l'emporte symboliquement hors de scène.

Le motif de la métamorphose en cadavre ou celui du protagoniste qui tombe dans les bras de la mort, devenue, comme dans le Purgatoire, son double dérisoire, sont devenus un recours si fréquent de la mise en scène de l'auto, que le dramaturge, souvent soucieux au cours de son œuvre d'opérer une sorte de distanciation (avant la lettre) avec ses procédés, va en profiter pour offrir une version comique du très pédagogique et très orthodoxe auto : dans la mojiganga Las visiones de la muerte, c'est le surgissement d'une troupe de comédiens ambulants qui allaient, avec leurs costumes, représenter un auto de ville en ville, qui détermine le quiproquo dans l'esprit quelque peu embrumé par le vin d'un caminante. Corps et Âme se battent ; il faut boire à la santé de la Mort, laquelle surgit avec sa faux et terrifie quelques gitans occupés à détrousser des Galiciens :

\section{Gitanos. - ¡Mueran todos! Mas, ;ay! que es Mi muerte la que yo encuentros.}

Il reste que la métamorphose brutale en cadavre ne saurait être représentée en elle-même ni pour elle-même : dans la mesure où il s'agit bien du topos du finis gloriae mundi, il faut, pour le dramaturge, développer un premier temps consacré à l'exposition de la gloire et du triomphe du pouvoir, lesquels seront bientôt réduits à l'état de cadavre dérisoire et mis en scène comme tels. Par ce biais, la métamorphose trouve un accès au drame historique.

Le Schisme dAngleterre offre, dans cette mesure, un cas particulièrement significatif : si la scène finale montre le triomphe d'Henri repenti et de sa fille Marie sur le trône royal, les didascalies précisent que les deux acteurs auront comme coussin pour leurs pieds le corps d'Anne Boleyn suppliciée (172a). Auparavant, toute la pièce a joué sur le motif du passage de la gloire la plus prestigieuse à la mort ou à la déchéance :

- Anne, dans sa première apparition lors du songe du roi, est qualifiée par Henri d'" ombre divine ": "Tente, sombra divina, imagen bella " (142a); le souvenir obsessionnel de cette image désirable déterminera le monarque à répudier Catherine d'Aragon et à épouser Anne Boleyn.

5. Pedro Calderón de la Barca, Entremeses, jácaras, mojigangas, éd. E. Rodríguez y A. Tordera, Castalia, coll. «Clásicos Castalia », 1982, p. 383. 
- Wolsey, favori du roi, se voit déjà promis au trône de saint Pierre ; la disgrâce royale le laisse soudain dépouillé :

\author{
Vivid, morid; que es penoso \\ estado llegarse a ver \\ un avaro sin poder \\ $y$ sin mando un ambicioso. (166b)
}

- Catherine d'Aragon, joue le rôle de la reine déchue et médite sur la brutale transformation de son destin qu' exprime, du moins à ses yeux, la métamorphose du palais en cercueil :

\author{
iAy, palacio proceloso \\ Mar de engaños y desdichas, \\ Ataúd con paños de oro, \\ Bóveda donde se guarda \\ La majestad vuelta en polvo! (163b)
}

L'aventure politique et amoureuse d'Anne Boleyn illustre l'échec du désir, qui entraîne la mort : en fait, toute une série de pièces montre le développement de passions "sans frein ", pour reprendre une métaphore habituelle de Calderón, et trouve son accomplissement dans le spectacle du cadavre du protagoniste, coupable de " concupiscence " et exposé comme tel, si l'on suit les didascalies. Libido sentiendi en ce qui concerne Amón, le violeur de sa sœur, poignardé et effondré sur la table d'un festin (fin de la deuxième journée de Los cabellos de Absalón, 849a) ; Libido dominandi en ce qui concerne Absalon, pendu par les cheveux à un arbre et percé de trois javelots (863b).

La mise en scène et l'exposition du cadavre du coupable constituent comme le nécessaire aboutissement de la tragédie, quand le protagoniste persévère dans son erreur, à la différence de Ludovico Enio (Purgatorio) ou d'Eusebio ( $\mathrm{La}$ devoción de la Cruz) qui sont pardonnés. Par cette disposition scénique, le lien est établi entre justice des hommes et justice divine, entre drama et auto : le supplicié est un damné en puissance.

Si El alcade de Zalamea est un drame de l'honneur centré sur la figure du labrador vieux chrétien qu'est Pedro Crespo, il ne faut pas pour autant oublier la fin du coupable violeur : jugé par les paysans du village en leur Conseil municipal, il est immédiatement exécuté, comme le précisent les didascalies : " on ouvre une porte et le Capitaine paraît, sur un siège, après le supplice du garrot " (569b). Même remarque concernant le finale de Las tres Justicias en una, où le coupable subit le châtiment de trois crimes différents, qui ne sont pas tous de son fait, mais, du moins, l'Ordre est-il rétabli par un roi qui se veut justicier avant tout (don Pèdre d'Aragon, dit le cruel). À l'égard de ce héros caldéronien, nouvel exemple de violent et de révolté sans limite, le monarque exerce lui-même la justice et soigne la mise en scène nocturne que découvrent les autres personnages : " on ouvre les portes qui seront celles du milieu du théâtre et l'on voit don Lope, sur un siège, dans la position d'un supplicié par 
le garrot, un papier dans sa main et deux chandelles à ses côtés » (709b). Il s'agit presque d'un tableau en clair-obscur, à la flamme d'une chandelle.

La métamorphose est, d'évidence, un phénomène spectaculaire, mais elle nourrit aussi la rhétorique caldéronienne et ses images demeurent au service d'une dialectique démonstrative dont l'exposition de la fin du corps est le pivot.

La transformation en cadavre vient fournir l'image exemplaire qui va servir de justification au vaste édifice de ce qui relève clairement de l'anthropologie jésuite : ce spectacle, en effet, est censé, comme pour les vanités, causer une prise de conscience brutale de la réalité de la mort, laquelle anéantit tout autant la chair que les espérances et les ambitions humaines. Dans la mesure où les jésuites (cf. les diverses prises de position lors de la querelle de auxiliis et l'opposition de la Compagnie aux dominicains) défendent la valeur d'un libre-arbitre capable de transformer une grâce suffisante en grâce efficace, le desengaño, qui doit suivre l'aperception de la métamorphose, peut s'avérer décisif pour entraîner le repentir et la " conversion " du pécheur, c'est-à-dire, au sens étymologique, le fait de se tourner (définitivement) vers Dieu par un acte de volonté et un repentir sans réserve.

À partir de l'instant de la conversion, la vie antérieure apparaît comme frappée d'irréalité, car tout entière fondée sur de fausses valeurs et des apparences : elle n'a alors pas plus de consistance et de réalité qu'un songe, pour celui qui s'éveillerait. On voit ainsi que la métamorphose du vivant en cadavre s'intègre également dans l'exploitation dramaturgique et rhétorique du topos ascétique de la vie comme songe :

\author{
Descanso del sueño hace \\ El hombre, jay Dios! sin que advierta \\ Que cuando duerme y despierta, \\ Cada día muere y nace; \\ Que vivo cadáver yace \\ Cada día, pues (rendida \\ La vida a un breve homicida \\ Que es su descanso), no advierte \\ Una lición que la Muerte \\ Le va estudiando a la vida ${ }^{6}$.
}

Le songe de la vie nous rappelle que le sommeil est une image de la mort et, inversement, que la mort est un sommeil dont on s'éveille pour la vérité absolue d'une vraie vie dans un au-delà de la mort. Comme la vie est un songe, elle est donc figure de la mort, ce qui légitime la métamorphose qui ne fait que mettre en évidence la réalité de la mort derrière l'apparence (fragile) de la vie : le gracioso de Amar después de la muerte, Alcouscous, sera chargé d'en tirer plaisamment la leçon :

\footnotetext{
¿Esto es dormer o morer?

Mas todo diz que es el mesmo,

$Y$ ser verdad, pues no sé

Si me muero o si me duermo (372b)
}

6. La Cena del rey Baltasar, 169b. 
peu de temps avant que son maître ne sorte du rêve de l'amour devant la réalité du cadavre de sa bien-aimée :

\section{[...] túmulo donde \\ La más dorada imagen, \\ Que iba siguiendo deidad, \\ Vino a conseguir cadáver? (375b)}

La fragilité de la vie, ainsi logiquement présentée comme toujours menacée par une irruption soudaine et imprévisible de la mort, commande le développement d'un certain nombre d'images et de figures de style qui sont chargées de représenter cette métamorphose virtuelle et perpétuellement menaçante du vivant. D'abord, elle détermine une définition oxymorique de l'homme en tant qu' " être-pour-la-mort ", pour reprendre les termes de l'existentialisme allemand issu de la réflexion heideggerienne : l'homme est alors décrit comme vivo cadáver, cadavre authentique sous les apparences du vivant. Les exemples de l'oxymore abondent sous la plume de Calderón, aussi bien dans les grands drames "métaphysiques " que dans les autos qui usent de la valeur pédagogique de la coexistence rhétorique et expressive des contraires. Comme autant d'avertissements prémonitoires, l'expression se retrouve au début du drame, soit lorsque Sigismond, émergeant de sa tour-prison, se définit comme "vivo cadáver" (502a) et "esqueleto vivo " (503a) devant Rosaura, soit quand le roi Egerius, dans sa frénésie, se présente "en brazos del sueño, vivo cadáver " (178a) ; la formule revient dans le finale du Prince constant, afin de souligner le sens de l'aventure de l'Infant, mort pauvre, malade, paralysé, réduit à mendier et véritable cadavre vivant dont la vue est insupportable à la belle Fénix (275a).

Finalement, si la réalité est semblable au songe, la vie est pareille à la mort, tout s'entremêle et les contraires coexistent en une métamorphose constante :

\footnotetext{
Que en las violencias del sueño, sepulcro tú de ti mismo, mueres vivo y vives muerto?.
}

Depuis la réflexion fondatrice de Jean Rousset sur la littérature baroque ${ }^{8}$, on sait que la rhétorique contemporaine de ce temps a usé et abusé des comparaisons de la vie humaine avec la bulle, la flamme ou la fleur, entendues comme symboles de la fragilité de notre condition. Pour un dramaturge toujours soucieux de donner forme allégorique à son propos et de représenter les concepts de sa philosophie religieuse $e^{9}$, la méditation désabusée sur la métamorphose de

\section{La Cena del rey Baltasar, 170b.}

8. Jean Rousset, La littérature de lâge baroque en France, Paris, José Corti, 1954.

9. Au point de faire parler ainsi l'Allégorie dans la loa de l'auto El Sacro Parnaso : "Je suis, si par des mots je me définis, la docte Allégorie, trope rhétorique, moi qui, parce qu'expressif, sous l'allusion à autre chose, fais signifier les propriétés en perspective, les accidents sous des apparences, car en donnant un corps au concept, j'anime même l'invisible " (traduction par 
la fleur, symbole de la vie qui allie beauté et caractère éphémère, offrait une possibilité d'amplification à laquelle il a eu fréquemment recours. En effet, la splendeur de la beauté comme l'éclat du pouvoir attirent les yeux, comme pour mieux leur infliger le spectacle tragique de leur soudaine dissolution, ainsi qu'au premier souffle d'un vent mauvais se métamorphose la fleur fragile ou la flamme vacillante dans la nuit.

De la façon la plus explicite, dans El Pleito matrimonial, la Vie portant une torche allumée vient entre Corps et Âme, pressés de jouir de la lumière du jour et les avertit :

\author{
Esta llama que arde fría \\ La vida de los dos es [...] \\ $Y$ Vida llama encendida \\ Que de los dos precedida \\ Vive tan sujeta al viento \\ Que de uno en otro momento \\ Dura lo que ha de durar. (79b)
}

Le lien entre la Fortune politique, soumise à la roue de la déesse, et le devenir de la fleur est particulièrement exploité par le personnage de Catherine d'Aragon dans le Schisme:

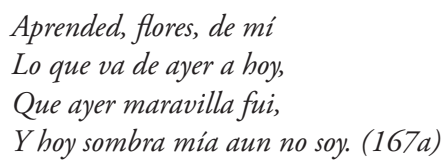

L'utilisation de ce réseau d'images centré sur la métamorphose et la destruction est peut-être encore plus systématique dans Le Prince Constant, drame qui commence par le chant des captifs célébrant la puissance du temps :

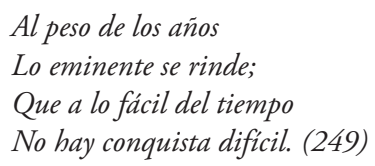

Or, le personnage de la belle Fénix, dont tout le monde célèbre la beauté, ne prend sens que pour être échangé finalement contre le cadavre du prince (" que en efecto esta hermosura / Precio de un muerto ha de ser ", 260a); le lien avec le motif du temps (en dehors du nom de l'héroïne, qui associe vie et mort) apparaît dès le début de la pièce, lorsque la princesse chante les fleurs ( e el jardin un mar de flores, / Y el mar un jardín de espumas ", 250a), condamnées à se faner, ainsi que l'exprime le célèbre sonnet des fleurs, prononcé par don Fernand, voué à l'esclavage en tant que jardinier de la belle :

Estas que fueron pompas y alegria,

Despertando el albor de la mañana,

A la tarde serán lástima vana,

Durmiendo en brazos de la noche fría. (266b)

l'auteur de l'article). 
À l'opposé de ces transformations dans le temps, la rare constance du prince n'en aura que plus d'éclat.

En définitive, c'est la femme qui rassemble toutes les caractéristiques de la métamorphose, devenant un sépulcre magnifique au dehors, apte à cacher un cadavre : tel est le sens des injures dont Amón accable sa sœur Tamar, après l'avoir violée (Cabellos de Absalón, 840a) :

\author{
Vete de aqui, salte afuera, \\ veneno en taza dorada, \\ sepulcro hermoso de fuera [...].
}

L'exploitation par Calderón du paradoxe spectaculaire de la métamorphose des prestiges du vivant en cadavre trouve peut-être son ultime signification dans l'acte même d'écrire pour un dramaturge qui a, au plus haut point, célébré la vie, la volonté de puissance, la révolte du vivant et la force destructrice des passions : telle est, du moins, la caractéristique commune des héros caldéroniens, de Sigismond à Ludovico Enio, en passant par Gómez Arias et Eusebio, et quelle que soit l'issue de leur parcours (salut ou perte du pécheur). On l'a souvent observé, il y a une étrange complaisance tout au long de la longue carrière de Calderón pour le personnage du révolté avide de jouir et de s'emparer de tous les biens du monde ; il y a pareillement une étrange complaisance pour le poète à célébrer la beauté du monde (et de la femme) et à en dénoncer simultanément l'illusion et la métamorphose dans le néant de la mort. Tel est peut-être, pour lui, le paradoxe fondateur de son œuvre : chanter le monde et reconnaître la nécessité de se taire devant l'évidence intellectuelle de sa vanité et de sa vacuité réelles. Psalle et sile : "chante et tais-toi », pour reprendre le titre d'une méditation poétique du dramaturge, dans laquelle on se plaît à reconnaître une confession. Entre les deux, l'expérience de la métamorphose conduit au silence, qui fait taire les passions et perce les apparences :

\author{
Es el silencio, un reservado archivo, \\ Donde la discreción tiene su asiento; [...] \\ Es contra el más colérico enemigo, \\ El más templado freno de la ira, \\ De la passión el más legal testigo, \\ Pues dice más que el que habla, el que suspira, \\ De la verdad tan familiar amigo, \\ Que a la simulación de la mentira \\ Se destiñe la tez, pues, quanto errante \\ Mintió la lengua, desmintió el semblante ${ }^{10}$.
}

10. Psalle et sile, reproduction en facsimilé de l'édition de 1662 du poème de Calderón, Valencia, Castalia, 1945, feuillets 5-6. 
